nothwendig beide treffen mufte. Эक jकioß, und bie Sugel fukr

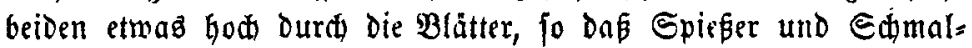
thier faft übereinander zufammen brachen und if nidjt nöthig latte, Diefelben now abjufangen.

$\mathfrak{B}$.

s. m.

\title{
Iiterarifde iseridjte.
}

№. 10.

Der wohlerfahrene Gäger. Für Gäger, Gagdfreunde und Gagd= beffizer von Dem Forfitmann uno Эäger Freiherrn Rubwig Şerdt non $\mathfrak{S u t t e n . ~ M i t ~ D r e i ~ f a r b i g e n ~} \mathfrak{T a f e l n}$. Mien 1863. T. Dirn= böđ'z $\mathfrak{B e r l a g . ~ R i . ~} 8^{0}$, IV und 188 S. ஒreiz 1 Thlr.

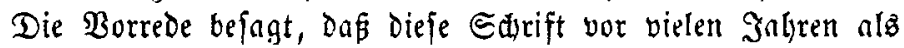
eine Borbereitung zu Der in Mariabrunn abjulegenten Pritfung aubigearbeitet wurbe. Sie habe Damnls annerfennung gefunden und werde nun Durch die fpätern (Erfahrungen Dez Berfonjes bereidfert Dem \$ublifum vorgelegt. \$s werden in Derfelten 100 Fragen aus Dem Bebiet Der Gagdriffenidaft aufgeftelt und beantmortet. Die

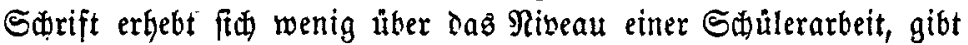
niffte Reues und wird mit einem $\mathfrak{T h a l e r}$ viel zu theuer bezaljlt.

№. 11.

$\mathfrak{B} i l d=$ und $\mathfrak{B}$ aiomannzbilder. Tagdicenen und Sdilderungen aub $\mathfrak{B a l d}$, Sefild und Şöhen. Mit SHuftrationen von $\mathfrak{B}$ erner, Rolb, Şildebrand u. $\mathfrak{A}$. 185 S. Keipzig (ohne Jahrebzakl). Berlag bon E. Bsiaferodt. \$retz 1 Thlt. 10 fgr.

Die 3nhl Der Büder, in weldyen die Ingd alz Stoff für unterbaltung herhalten muß, mefren fich mit Dem Jagbotilettantiz=

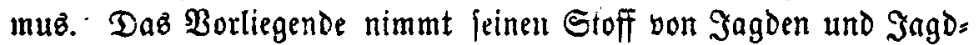
abenteuern auz beribiedenen Belttheilen mit mehr ober minder

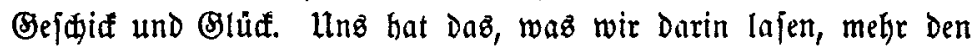

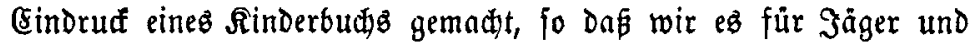
Sngoliebbaber nidit gerade empfeblem mödten. Die Slluftrationen find mäaig, mande ganz verunglỉatt.

v. $\mathfrak{B e r g}$.

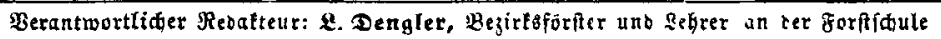
it Carlorufe. - Drut uno Berlag von E. Ed)meizerbart in Etuttgart. 